\title{
Adenocarcinoma Arising in an Ectopic Hamartomatous Thymoma with HER2 Overexpression
}

\author{
Harim Oh, Eojin Kim, Bokyung Ahn, Jeong Hyeon Lee, Youngseok Lee, Yang Seok Chae, Chul Hwan Kim, Yoo Jin Lee \\ Department of Pathology, Korea University Anam Hospital, Seoul, Korea
}

Ectopic hamartomatous thymoma (EHT) is a rare tumor which occurs almost exclusively in the supraclavicular or suprasternal area. Since first described by Smith and McClure in 1982, ${ }^{1}$ EHT has been reported in a total of 81 cases, which were mostly benign tumors. However, very rarely, a malignant tumor occurs in EHT, and only four cases have been reported. ${ }^{2-4}$ We report a case of a 52-year-old man with adenocarcinoma arising from EHT.

\section{CASE REPORT}

A 52-year-old man visited the hospital with a 1-year history of a supraclavicular mass which had grown to a size of $4 \mathrm{~cm}$ over the past $3-4$ months. The radiologic findings on contrastenhanced T1 weighted oropharynx magnetic resonance imaging showed a well-defined enhancing mass involving the left pectoralis major, suggesting a neurogenic tumor, complicated dermoid cyst or a reactive lymphadenopathy. Surgical removal was performed for diagnostic and therapeutic purposes. There has been no evidence of recurrence or metastasis in 6 months of follow up.

Grossly, the resected mass measured $3.1 \times 3.1 \times 1.8 \mathrm{~cm}$. On section, the cut surface showed a well-circumscribed, multilobulated, whitish-gray, heterogenous, solid mass with focal cystic changes (Fig. 1A).

Microscopically, the tumor could be largely divided into two portions. The periphery of the tumor displayed a haphazard blending of epithelial and spindle cells with focal adipose tissue

Received: May 20, 2019 Revised: June 4, 2019

Accepted: June 23, 2019

Corresponding Author: Yoo Jin Lee, MD

Department of Pathology, Korea University Anam Hospital, 73 Inchon-ro, Seongbuk-gu, Seoul 02841, Korea

Tel: +82-2-920-5595, Fax: +82-2-927-6576, E-mail: yujinn87@korea.ac.kr
(Fig. 1B). The dominant portion was spindle cell proliferation with moderate cellularity. These cells were arranged in a fascicular and storiform pattern with oval-to-tapered nuclei with eosinophilic cytoplasm. There was no evidence of significant atypia or mitotic activity. Some small lymphocytes were admixed with the spindle cell component. There were also epithelial islands consisting of elongated strands or anastomosing networks, and some cystic spaces with focal squamous differentiation. These islands were perceptibly merged into spindle cells. Small amounts of mature adipocytes were scattered in various portions of the tumor.

In the central portion of the tumor, areas of atypical glandular proliferation forming solid and cribriform architecture with an infiltrative edge were identified (Fig. 1C). The cells were oncocytoid with abundant, granular, eosinophilic cytoplasm, which are findings consistent with the features of apocrine cells and bearing some resemblance to ductal-type carcinoma. Unlike cells of the tumor periphery, the cells of the central portion had large pleomorphic nuclei with coarse chromatin and prominent nucleoli (Fig. 1D). Mitotic figures were identified and counted at an average of 3/high-power field, but atypical mitoses were not found.

On immunohistochemistry, both spindle cells and epithelial cells in the benign portion were reactive with pan-cytokeratin. The delicate spindle cells were positive for CD34, but negative for S-100. Both the spindle cells and the epithelial components of all areas of the tumor showed nuclear staining for androgen receptor (Fig. 1E).

EHT shows a biphasic pattern, so our differential diagnosis included other malignant lesions. Based on the CD34 and S100 immunohistochemistry results, we excluded synovial sarcoma and malignant peripheral nerve sheath tumor with epithelial differentiation. The tumor did not have chondromyxoid stroma, 
which can distinguish EHT from mixed tumor of the skin.

Notably, the dysplastic area showed complete and circumferential intense membrane staining of human epidermal growth factor receptor 2 (HER2) and scored $3+$ in accordance with the
HER2 analysis criteria for breast cancer (Fig. 1F). HER2 gene amplification was not performed.

Because the lesion had recently grown in size and the histological features showed a distinct malignant area with typical
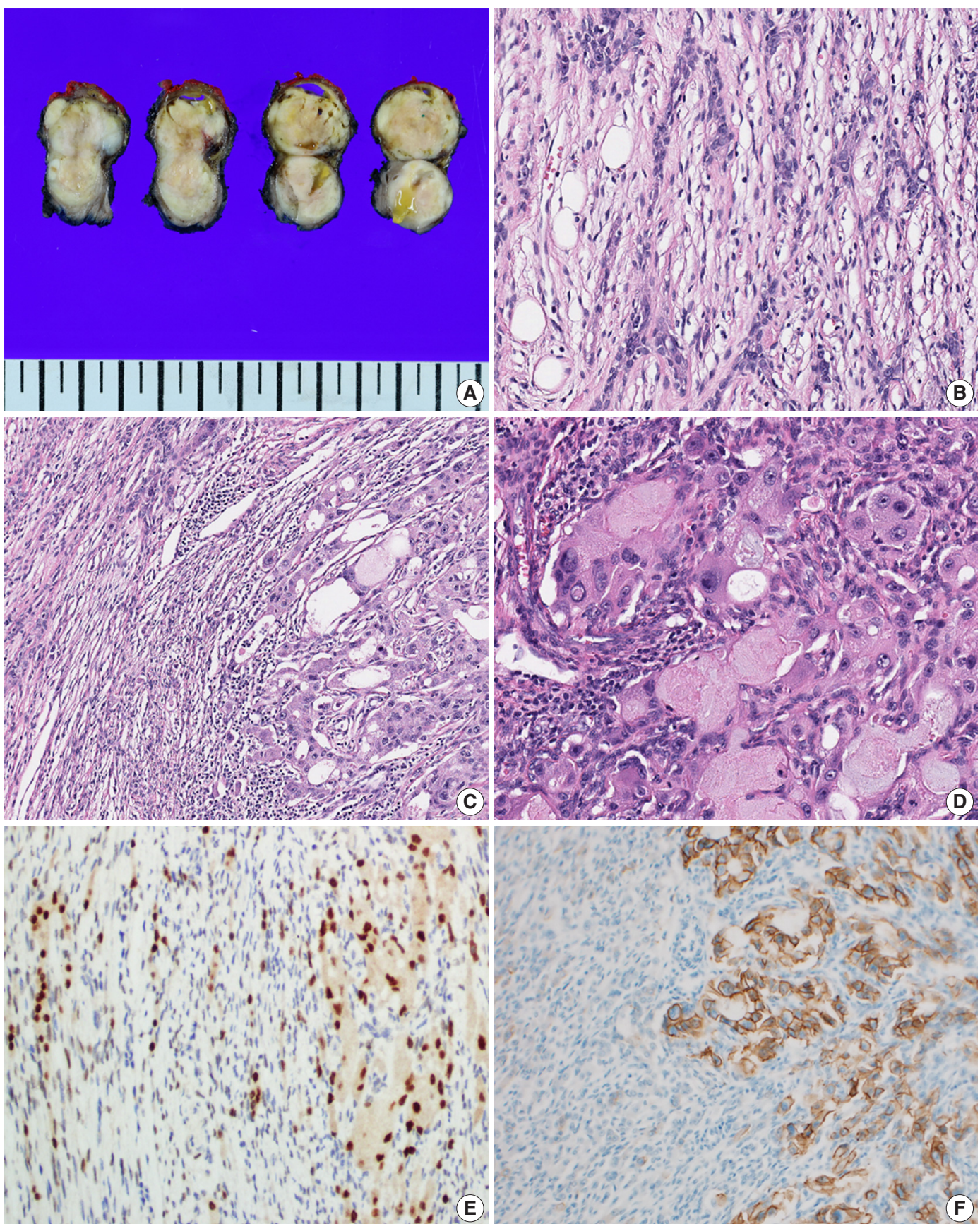

Fig. 1. Representative images of adenocarcinoma arising in ectopic hamartomatous thymoma (EHT). (A) Gross findings show a well-circumscribed, multi-lobulated, whitish-gray, heterogenous, solid mass with focal cystic changes. (B) The periphery of the tumor is composed of epithelial cells, spindle cells and adipose tissue, typical features of EHT. (C) The area of atypical glandular proliferation (right) is distinct from the background EHT (left). (D) The malignant components have large pleomorphic nuclei with prominent nucleoli and abundant eosinophilic cytoplasm. (E) Androgenic receptor immunostain shows nuclear positivity in both the benign and malignant areas. (F) HER2 overexpression is only identified in the malignant portion (right). 
EHT, we diagnosed this tumor as an adenocarcinoma arising in EHT.

\section{Ethics statement}

This report was approved by the Institutional Review Board of Korea University Anam Hospital (2018AN0340), and informed consent was waived.

\section{DISCUSSION}

EHT is a rare tumor, and only 81 cases have been reported thus far. It is generally known as benign, but there have been a few reports of malignancy. ${ }^{2-4}$ Three malignant cases had a portion of EHT with a distinct adenocarcinoma component. Consistent with these three cases, the present case demonstrated an area of atypical glandular proliferation with a punctuated cribriform or solid architecture where infiltration was identified, which was diagnosed as invasive adenocarcinoma arising in EHT.

According to the literature, a relatively high prevalence of HER2 overexpression was reported in ductal-type carcinoma ex pleomorphic adenoma, ${ }^{5}$ and HER2 overexpression was only restricted to the malignant portion. ${ }^{6}$ Also, the HER2 positivity was closely related to the aggressive behavior of carcinoma ex pleomorphic adenoma. ${ }^{5}$ These findings suggest that HER2 overexpression had an important role in the malignant transformation of pleomorphic adenoma and progression of carcinoma ex pleomorphic adenoma. Likewise, we could suggest that HER2 overexpression may explain the morphological similarity of the malignant area to ductal-type carcinoma (e.g., pleomorphic and oncocytic tumor cells), and HER2 might have a potential role in the malignant transformation of EHT.

One previous report associated EHT with the androgenic receptor. ${ }^{7}$ This implies that EHT is a tumor affected by androgenic stimuli and is further supported by the fact that EHT occurs exclusively in the adult male. ${ }^{7}$ Moreover, as the androgenic receptor was positive in both the benign and malignant areas of the tumor, we can suggest that the androgenic receptor is related to the occurrence of EHT.

To the best of our knowledge, this is the first case in which HER2 overexpression was found in a malignancy arising in EHT. Patients with HER2 overexpression have a therapeutic advantage because of the availability of targeted therapy, such as trastuzumab, once the mutation is confirmed. Therefore, identification of HER2 overexpression in adenocarcinoma arising from EHT could not only provide important information for understanding the pathogenesis of malignant transformation of EHT, but it can also indicate treatment with trastuzumab as a potential therapeutic target agent.

\section{ORCID}

Harim Oh: https://orcid.org/0000-0003-4904-4015

Eojin Kim: https://orcid.org/0000-0003-3111-6754

Bokyung Ahn: https://orcid.org/0000-0002-0229-2276

Jeong Hyeon Lee: https://orcid.org/0000-0003-2041-4617

Youngseok Lee: https://orcid.org/0000-0002-9762-4957

Yang Seok Chae: https://orcid.org/0000-0002-8801-0910

Chul Hwan Kim: https://orcid.org/0000-0003-2026-8824

Yoo Jin Lee: https://orcid.org/0000-0003-3830-7051

\section{Author Contributions}

Conceptualization: HO, YJL.

Data curation: EK, BA.

Investigation: $\mathrm{HO}, \mathrm{BA}$.

Project administration: JHL.

Resources: YL, YSC.

Supervision: YJL, CHK.

Validation: YL, YSC.

Visualization: EK, JHL.

Writing-Original Draft Preparation: HO.

Writing—Review \& Editing: YJL.

\section{Conflicts of Interest}

The authors declare that they have no potential conflicts of interest.

\section{Funding}

No funding to declare.

\section{REFERENCES}

1. Smith PS, McClure J. Unusual subcutaneous mixed tumour exhibiting adipose, fibroblastic, and epithelial components. J Clin Pathol 1982; 35: 1074-7.

2. Michal M, Zamecnik M, Gogora M, Mukensnabl P, Neubauer L. Pitfalls in the diagnosis of ectopic hamartomatous thymoma. Histopathology 1996; 29: 549-55.

3. Sato K, Thompson LDR, Miyai K, Kono T, Tsuda H. Ectopic hamartomatous thymoma: a review of the literature with report of new cases and proposal of a new name: biphenotypic branchioma. Head Neck Pathol 2018; 12: 202-9.

4. Michal M, Neubauer L, Fakan F. Carcinoma arising in ectopic 
hamartomatous thymoma: an ultrastructural study. Pathol Res Pract 1996; 192: 610-8.

5. Glisson B, Colevas AD, Haddad R, et al. HER2 expression in salivary gland carcinomas: dependence on histological subtype. Clin Cancer Res 2004; 10: 944-6.

6. Di Palma S, Skalova A, Vanieek T, Simpson RH, Starek I, Leivo I. Non-invasive (intracapsular) carcinoma ex pleomorphic adenoma: recognition of focal carcinoma by HER-2/neu and MIB1 immunohistochemistry. Histopathology 2005; 46: 144-52.

7. Weinreb I, O'Malley F, Ghazarian D. Ectopic hamartomatous thymoma: a case demonstrating skin adnexal differentiation with positivity for epithelial membrane antigen, androgen receptors, and BRST-2 by immunohistochemistry. Hum Pathol 2007; 38: 1092-5. 